\title{
About the Existence Results of Fractional Neutral Integrodifferential Inclusions with State-Dependent Delay in Fréchet Spaces
}

\author{
Selvaraj Suganya, ${ }^{1}$ Dumitru Baleanu, ${ }^{2,3}$ Siva Selvarasu, ${ }^{1}$ and Mani Mallika Arjunan' \\ ${ }^{1}$ Department of Mathematics, C. B. M. College, Kovaipudur, Coimbatore, Tamil Nadu 641 042, India \\ ${ }^{2}$ Department of Mathematics and Computer Sciences, Faculty of Art and Sciences, Cankaya University, 06530 Ankara, Turkey \\ ${ }^{3}$ Institute of Space Sciences, Magurele, Bucharest, Romania \\ Correspondence should be addressed to Dumitru Baleanu; dumitru@cankaya.edu.tr
}

Received 21 October 2015; Accepted 16 December 2015

Academic Editor: Hugo Leiva

Copyright (C) 2016 Selvaraj Suganya et al. This is an open access article distributed under the Creative Commons Attribution License, which permits unrestricted use, distribution, and reproduction in any medium, provided the original work is properly cited.

A recent nonlinear alternative for multivalued contractions in Fréchet spaces thanks to Frigon fixed point theorem consolidated with semigroup theory is utilized to examine the existence results for fractional neutral integrodifferential inclusions (FNIDI) with state-dependent delay (SDD). An example is described to represent the hypothesis.

\section{Introduction}

We are dealing in this paper with the existence of mild solutions for FNIDI with SDD in Fréchet spaces by making use of the fixed point theorem of Frigon [1, Corollary 3.5]. In Section 3 of this paper, we deliberate the neutral integrodifferential inclusions of fractional-order of the model

$$
\begin{aligned}
& \frac{d}{d t}\left[x(t)-\mathscr{G}\left(t, x_{\varrho\left(t, x_{t}\right)}\right)\right] \\
& \epsilon \int_{0}^{t} \frac{(t-s)^{\alpha-2}}{\Gamma(\alpha-1)} \mathscr{A}\left[x(s)-\mathscr{G}\left(s, x_{\varrho\left(s, x_{s}\right)}\right)\right] d s \\
& +\mathscr{F}\left(t, x_{\varrho\left(t, x_{t}\right)} \int_{0}^{t} e\left(t, s, x_{\varrho\left(s, x_{s}\right)}\right) d s\right), \\
& \text { a.e. } t \in \mathscr{I}=[0,+\infty),
\end{aligned}
$$

$$
x_{0}=\varsigma \in \mathscr{B} \text {, }
$$

wherein $1<\alpha<2$ and $\mathscr{A}: D(\mathscr{A}) \subset \mathbb{E} \rightarrow \mathbb{E}$ is the generator of an integral resolvent family characterized on a complex Banach space $(\mathbb{E},|\cdot|)$, the convolution integral within the equation is understood because of the Riemann-Liouville fractional integral (see [2]), $\mathscr{F}: \mathscr{I} \times \mathscr{B} \times \mathbb{E} \rightarrow \mathscr{P}(\mathbb{E})$ is a multivalued map, $(\mathscr{P}(\mathbb{E})$ is the family of nonempty subsets of $\mathbb{E}), e: \mathscr{I} \times \mathscr{I} \times \mathscr{B} \rightarrow \mathbb{E}, \mathscr{G}: \mathscr{I} \times \mathscr{B} \rightarrow \mathbb{E}$, and $\varrho: \mathscr{I} \times$ $\mathscr{B} \rightarrow(-\infty,+\infty)$ are apposite functions, and $\mathscr{B}$ is theoretical phase space axioms characterized in Section 2.

For almost any continuous function $x$ characterized on $(-\infty, b]$ and any $t \geq 0$, we designate by $x_{t}$ the part of $\mathscr{B}$ characterized by $x_{t}(\theta)=x(t+\theta)$ for $\theta \leq 0$. Now, $x_{t}(\cdot)$ speaks to the historical backdrop of the state from every $\theta \in(-\infty, 0]$ likely the current time $t$.

The notion of a fractional derivative plays an important role in numerous technological innovation and scientific disciplines as the statistical modeling of frameworks and procedures in numerous fields, case in point, physical science, chemical industry, aerodynamics, electrodynamics of complex medium, and so forth. For information, we recommend the readers to refer to the treatise of Abbas et al. [3], Baleanu et al. [4], Podlubny [5], Diethelm [6], Kilbas et al. [7], and Zhou [8] and the papers of fractional differential and integrodifferential systems [9-12] and impulsive fractional differential systems [13-15] and the references cited therein.

We recall that the fractional differential inclusions (FDI) occur in the mathematical modeling of specific models in financial aspects, optimal control, and so forth and are usually 
investigated by numerous writers; see, for instance, [16-18] and the references therein. Fractional equation with delay properties arises in several fields such as biological and physical ones with state-dependent delay or nonconstant delay. Nowadays, the existence results of mild solutions for such problems became very attractive and several researchers are working on it. Recently, several number of papers have been written on the fractional-order problems with statedependent delay [19-23] and the sources therein. In particular, in $[20,21]$, the authors analyzed the existence results for neutral differential systems with SDD in Banach spaces, whereas in $[19,22]$ the authors investigate the same type of problems with SDD and impulsive conditions by utilizing appropriate fixed point theorem. Also, the integrodifferential systems are experienced in numerous ranges of science, the place where it is imperative to deal with aftereffect or delay (e.g., control theory, biology, ecology, and medicine). Particularly, one dependably depicts a model which has inherited qualities by integrodifferential systems in implementation; see, for instance, [23-25].

The beginning stage of this work is reflected in [26-30]. Particularly, in [26], Agarwal et al. acquired the existence of mild solutions for FIDE of the structure

$$
\begin{aligned}
& x^{\prime}(t)=\int_{0}^{t} \frac{(t-s)^{\alpha-2}}{\Gamma(\alpha-1)} \mathscr{A} x(s) d s+f\left(t, x_{\varrho\left(t, x_{t}\right)}\right) \\
& x(0)=\varsigma \in \mathscr{B}, \\
& \text { a.e. } t \in \mathscr{I}=[0, b],
\end{aligned}
$$

in which $1<\alpha<2$ and $\mathscr{A}: D(\mathscr{A}) \subset \mathbb{X} \rightarrow \mathbb{X}$ is a linear densely described operator of sectorial kind on a complex Banach space $(\mathbb{X},|\cdot|)$, whereas, in [30], the authors establish the existence results for FIDI of the model

$$
\begin{gathered}
x^{\prime}(t)-\int_{0}^{t} \frac{(t-s)^{\alpha-2}}{\Gamma(\alpha-1)} \mathscr{A} x(s) d s \in \mathscr{F}\left(t, x_{\varrho\left(t, x_{t}\right)}\right), \\
\text { a.e. } t \in \mathscr{I}=[0, \infty), \\
x_{0}=\varsigma \in \mathscr{B},
\end{gathered}
$$

where $\alpha$ and $\mathscr{A}$ are the same as those mentioned in model (1).

The existence of mild solutions for the division of FNIDI with SDD in Fréchet spaces of the structure (1) is by all accounts an unread point. By utilizing a few speculations as a part of $[27,30]$, our desire here is to yield the existence results for the above model (1) utilizing a nonlinear alternative for multivalued contractions as of late created by Frigon [1, Corollary 3.5].

\section{Preliminary Notions}

Below, we briefly present the mathematical tools required in this paper.

Let $C([0, n], \mathbb{E}), n \in \mathbb{N}$, be the Banach space of all continuous functions from $\mathscr{I}_{n}=[0, n]$ into $\mathbb{E}$ making use of the standard norm

$$
\|x\|_{n}=\sup \{|x(t)|: 0 \leq t \leq n\} .
$$

Allow $B(\mathbb{E})$ to be the space of all bounded linear operators $\mathcal{N}: \mathbb{E} \rightarrow \mathbb{E}$, having the common supremum norm

$$
\|\mathcal{N}\|_{B(\mathbb{E})}=\sup \{|\mathcal{N}(x)|:|x|=1\} .
$$

A measurable function $x: \mathscr{I}_{n} \rightarrow \mathbb{E}$ is Bochner integrable if and only if $|x|$ is Lebesgue integrable (to get extra insights about Bochner integral, see the treatise of Yosida [31]).

Let $L^{1}\left(\mathscr{I}_{n}, \mathbb{E}\right)$ signify the Banach space of all measurable functions $x: \mathscr{I}_{n} \rightarrow \mathbb{E}$ which are Bochner integrable making use of the norm

$$
\|x\|_{L^{1}}=\int_{0}^{n}|x(t)| d t \quad \forall x \in L^{1}\left(\mathscr{I}_{n}, \mathbb{E}\right) .
$$

Recognize the space

$$
\begin{aligned}
& \mathscr{V}_{+\infty} \\
& \quad=\left\{x:(-\infty,+\infty) \longrightarrow \mathbb{E}:\left.x\right|_{\mathscr{I}} \in C(\mathscr{I}, \mathbb{E}), x_{0} \in \mathscr{B}\right\},
\end{aligned}
$$

where $\left.x\right|_{\mathscr{I}}$ is the restraint of $x$ to $\mathscr{I}$.

We expect that the phase space $\left(\mathscr{B},\|\cdot\|_{\mathscr{B}}\right)$ is a seminormed linear space of functions mapping $(-\infty, 0]$ into $\mathbb{E}$ and fulfilling the subsequent elementary adages as a result of Hale and Kato (find illustration in [32-34]).

$\left(P_{1}\right)$ If $x:(-\infty, n) \rightarrow \mathbb{E}$ is continuous on $\mathscr{I}_{n}$ and $x_{0} \epsilon$ $\mathscr{B}$, then for every $t \in \mathscr{I}_{n}$ the going hand in hand circumstances hold the following:

(i) $x_{t}$ is in $\mathscr{B}$;

(ii) $|x(t)| \leq H\left\|x_{t}\right\|_{\mathscr{B}}$;

(iii) $\left\|x_{t}\right\|_{\mathscr{B}} \leq \mathscr{D}_{1}(t) \sup \{|x(s)|: 0 \leq s \leq t\}+$ $\mathscr{D}_{2}(t)\left\|x_{0}\right\|_{\mathscr{B}}$, where $H>0$ is a constant and $\mathscr{D}_{1}(\cdot):[0,+\infty) \rightarrow[0,+\infty)$ is continuous, $\mathscr{D}_{2}(\cdot):[0,+\infty) \rightarrow[0,+\infty)$ is locally bounded, and $\mathscr{D}_{1}, \mathscr{D}_{2}$ are independent of $x(\cdot)$.

$\left(P_{2}\right)$ For function $x(\cdot)$ in $\left(P_{1}\right), x_{t}$ is a $\mathscr{B}$-valued continuous function on $\mathscr{I}_{n}$.

$\left(P_{3}\right)$ The space $\mathscr{B}$ is complete.

Designate $\mathscr{D}_{1}^{*}=\sup \left\{\mathscr{D}_{1}(t): t \in \mathscr{I}_{n}\right\}$ and $\mathscr{D}_{2}^{*}=$ $\sup \left\{\mathscr{D}_{2}(t): t \in \mathscr{I}_{n}\right\}$.

The next step is to review some known results from the fractional calculus.

The Laplace transformation of a function $f \in L_{\text {loc }}^{1}\left(\mathbb{R}^{+}, \mathbb{E}\right)$ is determined by

$$
\mathscr{L}(f)(\lambda)=\widehat{s}(\lambda)=\int_{0}^{\infty} e^{-\lambda t} f(t) d t, \quad \operatorname{Re}(\lambda)>\omega,
$$

if the integral is definitely convergent for $\operatorname{Re}(\lambda)>\omega$. With a specific end goal to give an operator hypothetical methodology, we review the subsequent definition [2].

Definition 1. Let $\mathscr{A}: D(\mathscr{A}) \subset \mathbb{E} \rightarrow \mathbb{E}$ be a closed and linear operator on a Banach space $\mathbb{E}$. One addresses $\mathscr{A}$ as the generator of an integral resolvent if one can find $\omega>0$ and 
a strongly continuous function $\mathcal{S}: \mathbb{R}^{+} \rightarrow B(\mathbb{E})$ to ensure that

$$
\left(\frac{1}{\widehat{s}(\lambda)} I-\mathscr{A}\right)^{-1} x=\int_{0}^{\infty} e^{-\lambda t} \mathcal{S}(t) x d t
$$

$\operatorname{Re} \lambda>\omega, x \in \mathbb{E}$

For this situation, $\delta(t)$ is known as the integral resolvent family produced by $\mathscr{A}$. For extra points of interest regarding this, we refer the reader to [35, Proposition 3.1 and Lemma 2.2].

Remark 2. The uniqueness and uniform continuity of the resolvent are long-familiar (see Benchohra and Litimein [30], Pruss [36]).

Before we complete this section, we display some longfamiliar outcomes from multivalued research.

Indicate the following:

$$
\begin{aligned}
\mathscr{P}(X) & =\{Y \subset X: Y \neq \emptyset\}, \\
\mathscr{P}_{\mathrm{cl}}(X) & =\{Y \in \mathscr{P}(X): Y \text { closed }\} \\
\mathscr{P}_{b}(X) & =\{Y \in \mathscr{P}(X): Y \text { bounded }\}, \\
\mathscr{P}_{\mathrm{cp}}(X) & =\{Y \in \mathscr{P}(X): Y \text { compact }\}, \\
\mathscr{P}_{\mathrm{cp}, c}(X) & =\{Y \in \mathscr{P}(X): Y \text { compact and convex }\} .
\end{aligned}
$$

Proposition 3 (see [37, Proposition III.4]). If $\Gamma_{1}$ and $\Gamma_{2}$ are compact valued measurable multifunctions, then the multifunction $t \rightarrow \Gamma_{1}(t) \cap \Gamma_{2}(t)$ is measurable. If $\left(\Gamma_{n}\right)$ is a sequence of compact valued measurable multifunctions, then $t \rightarrow \cap \Gamma_{n}(t)$ is measurable, and if $\overline{\cup \Gamma_{n}(t)}$ is compact, then $\overline{t \rightarrow \cup \Gamma_{n}(t)}$ is measurable.

Remark 4. The definitions of measurable, admissible contraction, metric space, and nonlinear alternative fixed point theorem [1, Corollary 3.5] are classical in multivalued analysis; hence, we keep off it.

Remark 5. For primary and surplus points on Fréchet spaces, we refer the reader to [30].

For each $x \in \mathscr{V}_{+\infty}$, specify the set of selections for $\mathscr{F}$ by

$$
\begin{aligned}
& S_{\mathscr{F}, x}=\left\{v \in L^{1}(\mathscr{I}, \mathbb{E}): v(t)\right. \\
& \quad \in \mathscr{F}\left(t, x_{\varrho\left(t, x_{t}\right)}, \int_{0}^{t} e\left(t, s, x_{\varrho\left(s, x_{s}\right)}\right) d s\right) \text { for a.e. } t \\
& \quad \in \mathscr{I}\} .
\end{aligned}
$$

For surplus points of benefit on multivalued maps, think about the treatise of Castaing and Valadier [37] and Graef et al. [38].

\section{The Main Results}

In this part, we prove the existence outcomes for the structure (1). To commence, we delineate the mild solution for the structure (1).

Definition 6. One affirms that the function $x:(-\infty,+\infty) \rightarrow$ $\mathbb{E}$ is a mild solution of the model (1) if $x(t)=\varsigma(t)$ for all $t \leq$ 0 , the constraint of $x(\cdot)$ to the period $[0, \infty)$ is continuous and one can find $v(\cdot) \in L^{1}(J, \mathbb{E})$, in a way that $v(t) \in \mathscr{F}(t$, $\left.x_{\varrho\left(t, x_{t}\right)}, \int_{0}^{t} e\left(t, s, x_{\varrho\left(s, x_{s}\right)}\right) d s\right)$ a.e. $t \in[0, \infty)$, and $x$ fulfills the consecutive integral equation:

$$
\begin{aligned}
x(t)= & \mathcal{S}(t)[\varsigma(0)-\mathscr{G}(0, \varsigma(0))]+\mathscr{G}\left(t, x_{\varrho\left(t, x_{t}\right)}\right) \\
& +\int_{0}^{t} \mathcal{S}(t-s) v(s) d s
\end{aligned}
$$

for every $t \in[0,+\infty)$.

Set

$$
\mathscr{R}\left(\varrho^{-}\right)=\{\varrho(s, \varsigma):(s, \varsigma) \in \mathscr{I} \times \mathscr{B}, \varrho(s, \varsigma) \leq 0\} .
$$

We generally expect that $\varrho: \mathscr{I} \times \mathscr{B} \rightarrow(-\infty, b]$ is continuous. Moreover, we make the subsequent assumption:

$\left(H_{\varsigma}\right)$ function $t \rightarrow \varsigma_{t}$ is continuous from $\mathscr{R}\left(\varrho^{-}\right)$into $\mathscr{B}$ and we can find a continuous and bounded function $L^{\varsigma}: \mathscr{R}\left(\varrho^{-}\right) \rightarrow(0, \infty)$ in a way that

$$
\left\|\varsigma_{t}\right\|_{\mathscr{B}} \leq L^{\varsigma}(t)\|\varsigma\|_{\mathscr{B}} \quad \text { for every } t \in \mathscr{R}\left(\varrho^{-}\right) \text {. }
$$

Lemma 7 (see [21, Lemma 3.1]). If $x:(-\infty, b] \rightarrow \mathbb{X}$ is $a$ function to ensure that $x_{0}=\varsigma$, then

$$
\begin{aligned}
\left\|x_{s}\right\|_{\mathscr{B}} \leq & \left(\mathscr{D}_{2}^{*}+L^{\varsigma}\right)\|\varsigma\|_{\mathscr{B}} \\
+ & \mathscr{D}_{1}^{*} \sup \{|x(\theta)|: \theta \in[0, \max \{0, s\}]\}, \\
& \quad s \in \mathscr{R}\left(\varrho^{-}\right) \cup \mathscr{I}_{n},
\end{aligned}
$$

where $L^{\varsigma}=\sup _{t \in \mathscr{R}\left(\varrho^{-}\right)} L^{\varsigma}(t)$.

The successive hypotheses will be required in whatever is left of this paper.

(H1) The solution operator $\delta(t)_{t \in \mathscr{I}}$ is compact for $t \geq 0$, and we can find $\mathscr{M}>0$ in a way that

$$
\|\mathcal{S}(t)\|_{B(\mathbb{E})} \leq \mathscr{M} \text { for every } t \in \mathscr{I} .
$$

(i) The multivalued map $\mathscr{F}: \mathscr{I} \times \mathscr{B} \times \mathbb{E} \rightarrow$ $\mathscr{P}_{\text {cp,c }}(\mathbb{E})$ is Carathéodory and there is certainly function $\vartheta \in L_{\text {loc }}^{1}\left(\mathscr{I}, \mathbb{R}^{+}\right)$and a continuous nondecreasing function $\circ: \mathbb{R}^{+} \rightarrow(0, \infty)$ in a way that

$$
|\mathscr{F}(t, u, y)| \leq \vartheta(t) \curvearrowright\left(\|u\|_{\mathscr{B}}+\|y\|\right),
$$

$$
\text { for every }(t, u, y) \in \mathscr{I} \times \mathscr{B} \times \mathbb{E} \text {. }
$$


(ii) For all $\mathscr{R}>0$, we can find $\wp_{\mathscr{R}} \in L_{\text {loc }}^{1}\left(\mathscr{I}, \mathbb{R}^{+}\right)$to ensure that

$$
\begin{aligned}
& H_{d}(\mathscr{F}(t, u, y), \mathscr{F}(t, \bar{u}, \bar{y})) \\
& \quad \leq \wp_{\mathscr{R}}(t)\left(\|u-\bar{u}\|_{\mathscr{B}}+\|y-\bar{y}\|\right),
\end{aligned}
$$

in which $t \in \mathscr{I}$, in addition to, for all $u, \bar{u} \in \mathscr{B}$ in conjunction with $\left\{\|u\|_{\mathscr{B}},\|\bar{u}\|_{\mathscr{B}}\right\} \leq \mathscr{R}, y, \bar{y} \in \mathbb{E}$ joined with

$$
d(0, \mathscr{F}(t, 0,0)) \leq \wp_{\mathscr{R}}(t) \quad \text { a.e. } t \in \mathscr{I} .
$$

(H3)

(i) There is a function $m \in L_{\text {loc }}^{1}\left(\mathscr{I}, \mathbb{R}^{+}\right)$and a continuous nondecreasing function $\Omega: \mathbb{R}^{+} \rightarrow$ $(0, \infty)$ to ensure that

$|e(t, s, u)| \leq m(s) \Omega\left(\|u\|_{\mathscr{B}}\right)$

$$
\forall(t, s, u) \in \mathscr{I} \times \mathscr{I} \times \mathscr{B} .
$$

(ii) There is a constant $C_{1}>0$ in a way that

$$
\begin{aligned}
\left|\int_{0}^{t}[e(t, s, u)-e(t, s, \bar{u})] d s\right| & \leq C_{1}\|u-\bar{u}\|_{\mathscr{B}}, \\
& \text { for }(t, s) \in \mathscr{I},(u, \bar{u}) \in \mathscr{B} .
\end{aligned}
$$

(H4)

(i) Function $\mathscr{G}(t, \cdot)$ is continuous on $\mathscr{I}$, and there are certainly positive constants $c_{1}, c_{2}$ in a way that

$|\mathscr{G}(t, u)| \leq c_{1}\|u\|_{\mathscr{B}}+c_{2}$, for every $(t, u) \in \mathscr{I} \times \mathscr{B}$. (ii) For every $\mathscr{R}>0$, there is a function $\widetilde{\wp}_{\mathscr{R}}(t) \epsilon$ $L_{\text {loc }}^{1}\left(\mathscr{I}, \mathbb{R}^{+}\right)$in a way that

$$
\begin{aligned}
|\mathscr{G}(t, u)-\mathscr{G}(t, \bar{u})| \leq \widetilde{\wp}_{\mathscr{R}}(t)\|u-\bar{u}\|_{\mathscr{B}}, \\
\quad t \in \mathscr{I}, u, \bar{u} \in \mathscr{B} \text { with }\left\{\|u\|_{\mathscr{B}},\|\bar{u}\|_{\mathscr{B}}\right\} \leq \mathscr{R} .
\end{aligned}
$$

For each $n \in \mathbb{N}$, we delineate, in $\mathscr{V}_{+\infty}$, the family of seminorms by

$$
\|x\|_{n}=\sup \left\{e^{-\tau L_{n}^{*}(t)}|x(t)|: t \in[0, n]\right\}
$$

in which $L_{n}^{*}(t)=\int_{0}^{t} \wp_{n}^{*}(s) d s, \wp_{n}^{*}(t)=\max \left\{\widetilde{\wp}_{n}(t) \mathscr{D}_{1}^{*}, \bar{\wp}(t)\right\}$, $\bar{\wp}(t)=M_{\wp_{n}}(t)\left(1+C_{1}\right) \mathscr{D}_{1}^{*}$, and $\wp_{n}^{*}=\sup \left\{\wp_{n}^{*}(t): t \in[0, n]\right\}$ and accept that $\left(\wp_{n}^{*}+1 / \tau\right)<1$ and $\wp_{n}, \widetilde{\wp}_{n}$ are a function from $(\mathrm{H} 2)(\mathrm{ii})$ and (H4)(ii) appropriately.

Theorem 8. Expect that $(\mathrm{H} 1)-(\mathrm{H} 4)$ and $\left(\mathrm{H}_{c}\right)$ hold, and believe that $\mu=1-c_{1} \mathscr{D}_{1}^{*}>0$ and

$$
\int_{C}^{+\infty} \frac{d s}{\delta(s)+\Omega(s)}>\int_{0}^{n} \nu(s) d s \quad \text { for } n \in \mathbb{N}
$$

in which $C=c_{n}+\left(\mathscr{D}_{1}^{*} / \mu\right)\left[\mathscr{M}|\mathscr{G}(0, \varsigma(0))|+c_{1} c_{n}+c_{2}\right]$. At that point, model (1) has a mild solution on $(-\infty,+\infty)$.

Proof. We will transmute the structure (1) into a fixed point problem. Recognize the multivalued operator $\Upsilon: \mathscr{V}_{+\infty} \rightarrow$ $\mathscr{P}\left(\mathscr{V}_{+\infty}\right)$ specified by $\Upsilon(h)=\left\{h \in \mathscr{V}_{+\infty}\right\}$ with

$$
h(t)= \begin{cases}\varsigma(t), & t \leq 0 ; \\ \mathcal{S}(t)[\varsigma(0)-\mathscr{G}(0, \varsigma(0))]+\mathscr{G}\left(t, x_{\varrho\left(t, x_{t}\right)}\right)+\int_{0}^{t} \mathcal{S}(t-s) v(s) d s, & t \in \mathscr{I},\end{cases}
$$

where $v \in S_{\mathscr{F}, x_{Q\left(s, x_{s}\right)}}$. For $\varsigma \in \mathscr{B}$, we express function $y(\cdot)$ : $(-\infty,+\infty) \rightarrow \mathbb{E}$ by

$$
y(t)= \begin{cases}\varsigma(t), & t \leq 0 \\ \mathcal{S}(t) \varsigma(0), & t \in \mathscr{I}\end{cases}
$$

and then $y_{0}=\varsigma$. For every function $z \in \mathscr{V}_{+\infty}$ with $z_{0}=0$, we designate by $\widetilde{z}$ the function clear by

$$
\widetilde{z}(t)= \begin{cases}0, & t \leq 0 \\ z(t), & t \in \mathscr{I} .\end{cases}
$$

If $x(\cdot)$ fulfills (12), we are able to decompose it as $x(t)=z(t)+$ $y(t), t \in \mathscr{I}$, which suggests that $x_{t}=z_{t}+y_{t}$, for each $t \in \mathscr{I}$, and also the function $z(\cdot)$ fulfills

$$
\begin{aligned}
z(t)= & \mathscr{G}\left(t, z_{\varrho\left(t, z_{t}+y_{t}\right)}+y_{\varrho\left(t, z_{t}+y_{t}\right)}\right)-\mathcal{S}(t) \mathscr{G}(0, \varsigma(0)) \\
& +\int_{0}^{t} \mathcal{S}(t-s) v(s) d s, \quad t \in \mathscr{I}
\end{aligned}
$$

where $v(s) \in \mathcal{S}_{\mathscr{F}, z_{\varrho\left(s, z_{s}+y_{s}\right)}+y_{\varrho\left(s, z_{s}+y_{s}\right)}}$.

Let $\mathscr{V}_{+\infty}^{0}=\left\{z \in \mathscr{V}_{+\infty}: z(0)=0 \in \mathscr{B}\right\}$. For any $z \in$ $\mathscr{V}_{+\infty}^{0}$, we sustain

$$
\begin{aligned}
\|z\|_{+\infty} & =\sup \{|z(s)|: 0 \leq s<+\infty\}+\left\|z_{0}\right\|_{\mathscr{B}} \\
& =\sup \{|z(s)|: 0 \leq s<+\infty\} .
\end{aligned}
$$


Along these lines, $\mathscr{V}_{+\infty}^{0}$ is a Banach space with the norm $\|\cdot\|_{+\infty}$. We delimit the operator $\bar{Y}: \mathscr{V}_{+\infty}^{0} \rightarrow \mathscr{P}\left(B_{+\infty}^{0}\right)$ by $\bar{\Upsilon}(z)=\left\{h \in \mathscr{V}_{+\infty}^{0}\right\}$ with

$$
\begin{aligned}
h(t)= & \mathscr{G}\left(t, z_{\varrho\left(t, z_{t}+y_{t}\right)}+y_{\varrho\left(t, z_{t}+y_{t}\right)}\right)-\mathcal{S}(t) \mathscr{G}(0, \varsigma(0)) \\
& +\int_{0}^{t} \mathcal{S}(t-s) v(s) d s, \quad t \in \mathscr{F}
\end{aligned}
$$

where $v(s) \in S_{\mathscr{F}, z_{\rho\left(s, z_{s}+y_{s}\right)}+y_{\varrho\left(s, z_{s}+y_{s}\right)}}$.

It is vindicated that the operator $Y$ has a fixed point if and only if $\bar{\Upsilon}$ has a fixed point. As a result, let us demonstrate that $\bar{\Upsilon}$ has a fixed point $z \in \mathscr{V}_{+\infty}^{0}$.

Remark 9. (i) By condition $\left(P_{1}\right)$ and Lemma 7 in the above discussion, we have the subsequent estimates:

$$
\begin{aligned}
&\left\|z_{\varrho\left(s, z_{s}+y_{s}\right)}+y_{\varrho\left(s, z_{s}+y_{s}\right.}\right\|_{\mathscr{B}} \\
& \leq\left\|z_{\varrho\left(s, z_{s}+y_{s}\right)}\right\|_{\mathscr{B}}+\left\|y_{\varrho\left(s, z_{s}+y_{s}\right)}\right\|_{\mathscr{B}} \\
& \leq \mathscr{D}_{1}^{*}|z(s)|+\left(\mathscr{D}_{2}^{*}+L^{\varsigma}\right)\left\|z_{0}\right\|_{\mathscr{B}}+\mathscr{D}_{1}^{*}|y(s)| \\
&+\left(\mathscr{D}_{2}^{*}+L^{\varsigma}\right)\|\varsigma\|_{\mathscr{B}} \\
& \leq \mathscr{D}_{1}^{*}|z(s)|+\mathscr{D}_{1}^{*} \mathscr{M} H\|\varsigma\|_{\mathscr{B}}+\left(\mathscr{D}_{2}^{*}+L^{\varsigma}\right)\|\varsigma\|_{\mathscr{B}} \\
& \leq \mathscr{D}_{1}^{*}|z(s)|+\left(\mathscr{D}_{2}^{*}+L^{\varsigma}+\mathscr{D}_{1}^{*} \mathscr{M} H\right)\|\varsigma\|_{\mathscr{B}} \\
& \leq \mathscr{D}_{1}^{*}|z(s)|+c_{n},
\end{aligned}
$$

where $c_{n}=\left(\mathscr{D}_{2}^{*}+L^{\varsigma}+\mathscr{D}_{1}^{*} \mathscr{M} H\right)\|\varsigma\|_{\mathscr{B}}$.

(ii)

$$
\begin{aligned}
& |v(t)-\bar{v}(t)| \leq \wp_{n}(t)\left[\left\|z_{\varrho\left(t, z_{t}+y_{t}\right)}-\bar{z}_{\varrho\left(t, \bar{z}_{t}+y_{t}\right)}\right\|_{\mathscr{B}}\right. \\
& \left.+C_{1}\left\|z_{\varrho\left(t, z_{t}+y_{t}\right)}-\bar{z}_{\varrho\left(t, \bar{z}_{t}+y_{t}\right)}\right\|_{\mathscr{B}}\right] \leq \wp_{n}(t) \\
& \cdot\left[\mathscr{D}_{1}^{*}|z(t)-\bar{z}(t)|+C_{1} \mathscr{D}_{1}^{*}|z(t)-\bar{z}(t)|\right] \leq \wp_{n}(t) \\
& \cdot\left(1+C_{1}\right) \mathscr{D}_{1}^{*}|z(t)-\bar{z}(t)| ;
\end{aligned}
$$

(iii)

$$
\begin{aligned}
& |h(t)-\bar{h}(t)| \leq \mid \mathscr{G}\left(t, z_{\varrho\left(t, z_{t}+y_{t}\right)}+y_{\varrho\left(t, z_{t}+y_{t}\right)}\right) \\
& \quad-\mathscr{G}\left(t, \bar{z}_{\varrho\left(t, \bar{z}_{t}+y_{t}\right)}+y_{\varrho\left(t, \bar{z}_{t}+y_{t}\right)}\right) \mid \\
& \quad+\int_{0}^{t}\|\mathcal{S}(t-s)\|_{B(\mathbb{E})}|v(s)-\bar{v}(s)| d s \leq \widetilde{\wp}_{n}(t) \\
& \cdot \mathscr{D}_{1}^{*}|z(t)-\bar{z}(t)| \\
& +\mathscr{M}_{0}^{t} \wp_{n}(s)\left(1+C_{1}\right) \mathscr{D}_{1}^{*}|z(s)-\bar{z}(s)| d s \\
& \quad \leq \widetilde{\wp}_{n}(t) \mathscr{D}_{1}^{*}|z(t)-\bar{z}(t)| \\
& \quad+\int_{0}^{t} \bar{\wp}_{n}(s)|z(s)-\bar{z}(s)| d s \leq\left[\widetilde{\wp}_{n}(t) \mathscr{D}_{1}^{*} e^{\tau L_{n}^{*}(t)}\right] \\
& \quad \cdot\left[e^{-\tau L_{n}^{*}(t)}|z(t)-\bar{z}(t)|\right]
\end{aligned}
$$

$$
\begin{aligned}
& +\int_{0}^{t}\left[\bar{\wp}_{n}(s) e^{\tau L_{n}^{*}(s)}\right]\left[e^{-\tau L_{n}^{*}(s)}|z(s)-\bar{z}(s)|\right] d s \\
& \leq \wp_{n}^{*}(t) e^{\tau L_{n}^{*}(t)}\|z-\bar{z}\|_{n} \\
& +\int_{0}^{t}\left[\wp_{n}^{*}(s) e^{\tau L_{n}^{*}(s)}\right]\|z-\bar{z}\|_{n} d s \leq\left(\wp_{n}^{*}+\frac{1}{\tau}\right) \\
& \cdot e^{\tau L_{n}^{*}(t)}\|z-\bar{z}\|_{n} .
\end{aligned}
$$

Presenting $n \in \mathbb{N}, z$ is ought to be a solution of the inclusion $z \in \lambda \bar{Y}(z)$ for many $\lambda \in(0,1)$ and there may be $v \in$ $S_{\mathscr{F}, z_{\ell\left(s, z_{s}+y_{s}\right)}+y_{\varrho\left(s, z_{s}+y_{s}\right)}}$ in a way that, for any $t \in \mathscr{I}_{n}$, we maintain

$$
\begin{aligned}
& |z(t)| \leq\|\mathcal{S}(t)\|_{B(\mathbb{E})}|\mathscr{G}(0, \varsigma(0))|+\mid \mathscr{G}\left(t, z_{\varrho\left(t, z_{t}+y_{t}\right)}\right. \\
& \left.+y_{\varrho\left(t, z_{t}+y_{t}\right)}\right)\left|+\int_{0}^{t}\|\mathcal{S}(t-s)\|_{B(\mathbb{E})}\right| \mathscr{F}\left(s, z_{\varrho\left(s, z_{s}+y_{s}\right)}\right. \\
& +y_{\varrho\left(s, z_{s}+y_{s}\right)} \\
& \left.\quad \int_{0}^{s} e\left(s, \tau, z_{\varrho\left(\tau, z_{\tau}+y_{\tau}\right)}+y_{\varrho\left(\tau, z_{\tau}+y_{\tau}\right)}\right) d \tau\right) \mid d s \\
& \quad \leq \mathscr{M}|\mathscr{G}(0, \varsigma(0))|+c_{1}\left\|z_{\varrho\left(t, z_{t}+y_{t}\right)}+y_{\varrho\left(t, z_{t}+y_{t}\right)}\right\|_{\mathscr{B}} \\
& \quad+c_{2}+\mathscr{M} \int_{0}^{t} \mathcal{\vartheta}(s) \curvearrowright \curvearrowright\left(\left\|z_{\varrho\left(s, z_{s}+y_{s}\right)}+y_{\varrho\left(s, z_{s}+y_{s}\right)}\right\|_{\mathscr{B}}\right. \\
& \left.\quad+\int_{0}^{s} m(\tau) \Omega\left(\left\|z_{\varrho\left(s, z_{s}+y_{s}\right)}+y_{\varrho\left(s, z_{s}+y_{s}\right)}\right\|_{\mathscr{B}}\right) d \tau\right) d s .
\end{aligned}
$$

From Remark 9(i), we have

$$
\begin{aligned}
& |z(t)| \leq \mathscr{M}|\mathscr{G}(0, \varsigma(0))|+c_{1} \mathscr{D}_{1}^{*}|z(t)|+c_{1} c_{n}+c_{2} \\
& +\quad \mathscr{M} \int_{0}^{t} \vartheta(s) \diamond\left(\mathscr{D}_{1}^{*}|z(s)|+c_{n}\right. \\
& \left.\quad+\int_{0}^{s} m(\tau) \Omega\left(\mathscr{D}_{1}^{*}|z(s)|+c_{n}\right) d \tau\right) d s \\
& \quad \leq \frac{1}{\mu}\left[\mathscr{M}|\mathscr{G}(0, \varsigma(0))|+c_{1} c_{n}+c_{2}\right]+\frac{\mathscr{M}}{\mu} \int_{0}^{t} \vartheta(s) \\
& . \diamond\left(\mathscr{D}_{1}^{*}|z(s)|+c_{n}\right. \\
& \left.+\int_{0}^{s} m(\tau) \Omega\left(\mathscr{D}_{1}^{*}|z(s)|+c_{n}\right) d \tau\right) d s .
\end{aligned}
$$

Thus,

$$
\begin{gathered}
\mathscr{D}_{1}^{*}|z(t)|+c_{n} \leq c_{n}+\frac{\mathscr{D}_{1}^{*}}{\mu}\left[\mathscr{M}|\mathscr{G}(0, \varsigma(0))|+c_{1} c_{n}\right. \\
\left.+c_{2}\right]+\frac{\mathscr{M} \mathscr{D}_{1}^{*}}{\mu} \int_{0}^{t} \vartheta(s) \circ\left(\mathscr{D}_{1}^{*}|z(s)|+c_{n}\right. \\
\left.\quad+\int_{0}^{s} m(\tau) \Omega\left(\mathscr{D}_{1}^{*}|z(s)|+c_{n}\right) d \tau\right) d s .
\end{gathered}
$$


We conceive function $\beta$ characterized by

$$
\beta(t)=\sup \left\{\mathscr{D}_{1}^{*}|z(s)|+c_{n}: 0 \leq s \leq b\right\},
$$

$$
0 \leq t<+\infty \text {. }
$$

Permit $t^{*} \in[0, t]$ in a way that $\beta(t)=\mathscr{D}_{1}^{*}\left|z\left(t^{*}\right)\right|+c_{n}\|\varsigma\|_{\mathscr{B}}$. Because of the aforementioned inequality, we maintain, for $t \in \mathscr{I}_{n}$,

$$
\begin{aligned}
& \beta(t) \leq c_{n}+\frac{\mathscr{D}_{1}^{*}}{\mu}\left[\mathscr{M}|\mathscr{G}(0, \varsigma(0))|+c_{1} c_{n}+c_{2}\right] \\
& +\frac{\mathscr{M} \mathscr{D}_{1}^{*}}{\mu} \\
& \quad \cdot \int_{0}^{t} \vartheta(s) \curvearrowright\left(\beta(s)+\int_{0}^{s} m(\tau) \Omega(\beta(\tau)) d \tau\right) d s .
\end{aligned}
$$

Allow us to occupy the right-hand part of the overhead inequality as $w(t)$. Then, we sustain $\beta(t) \leq w(t)$ for all $t \in$ $[0, n]$. Through the significance of $v$, we get

$$
w(0)=c_{n}+\frac{\mathscr{D}_{1}^{*}}{\mu}\left[\mathscr{M}|\mathscr{G}(0, \varsigma(0))|+c_{1} c_{n}+c_{2}\right]=C .
$$

This leads us to the accompanying inequality for $t \in \mathscr{I}_{n}$,

$$
\begin{aligned}
& w(t) \leq c_{n}+\frac{\mathscr{D}_{1}^{*}}{\mu}\left[\mathscr{M}|\mathscr{G}(0, \varsigma(0))|+c_{1} c_{n}+c_{2}\right] \\
& +\frac{\mathscr{M} \mathscr{D}_{1}^{*}}{\mu} \\
& \quad \cdot \int_{0}^{t} \vartheta(s) \curvearrowright\left(w(s)+\int_{0}^{s} m(\tau) \Omega(w(\tau)) d \tau\right) d s,
\end{aligned}
$$

where

$$
\begin{aligned}
& w^{\prime}(t) \\
& \quad \leq \frac{\mathscr{M}_{1}^{*}}{\mu} \vartheta(t) \curvearrowright\left(w(t)+\int_{0}^{t} m(s) \Omega(w(s)) d s\right) .
\end{aligned}
$$

Next, we weigh the function

$$
\omega(t)=w(t)+\int_{0}^{t} m(s) \Omega(w(s)) d s .
$$

Then we bring forth $\omega(0)=w(0)=C$ and $w(t) \leq \omega(t)$ for all $t \in \mathscr{I}_{n}$. Applying the nondecreasing character of $\odot$, we receive

$$
\begin{aligned}
\omega^{\prime}(t) & =w^{\prime}(t)+m(t) \Omega(w(t)) \\
& \leq \frac{\mathscr{M}_{1}^{*}}{\mu} \vartheta(t) \curvearrowright(\omega(t))+m(t) \Omega(\omega(t)),
\end{aligned}
$$

a.e. $t \in \mathscr{I}_{n}$.

We characterize the function $\nu(t)=\max \left\{\left(\mathscr{M} \mathscr{D}_{1}^{*} / \mu\right) \vartheta(t)\right.$, $m(t)\}, t \in \mathscr{I}_{n}$, which suggests that

$$
\frac{\omega^{\prime}(t)}{\delta(\omega(t))+\Omega(\omega(t))} \leq v(t)
$$

From condition (25), we acquire

$$
\begin{gathered}
\int_{\omega(0)=C}^{\omega(t)} \frac{d s}{\delta(s)+\Omega(s)} \leq \int_{0}^{t} \nu(s) d s \leq \int_{0}^{n} \nu(s) d s \\
\quad<\int_{C}^{+\infty} \frac{d s}{\curvearrowright(s)+\Omega(s)} .
\end{gathered}
$$

Subsequently, for every $t \in \mathscr{I}_{n}$, we have a constant $\Lambda_{n_{*}}$ in a way that $\omega(t) \leq \Lambda_{n_{*}}$ and, consequently, $\beta(t) \leq \Lambda_{n_{*}}$. Due to the fact that $\|z\|_{n} \leq \beta(t)$, we certainly have $\|z\|_{n} \leq \Lambda_{n_{*}}$. Fix

$$
\mathcal{U}=\left\{z \in \mathscr{V}_{+\infty}^{0}: \sup _{0 \leq t \leq n}|z(t)| \leq \Lambda_{n_{*}}+1, \forall n \in \mathbb{N}\right\} .
$$

Evidently, $\mathcal{U}$ is a closed subset of $\mathscr{V}_{+\infty}^{0}$. We should demonstrate that $\bar{\Upsilon}: \overline{\mathscr{U}} \rightarrow \mathscr{P}\left(\mathscr{V}_{+\infty}^{0}\right)$ is a contraction and an admissible operator. Initially, we evaluate that $\bar{Y}$ is a contraction. In fact, consider $z, \bar{z} \in \mathscr{V}_{+\infty}^{0}$ and $h \in \bar{\Upsilon}(z)$. Then, there may be $v(t) \in \mathscr{F}\left(t, z_{\varrho\left(t, z_{t}+y_{t}\right)}+y_{\varrho\left(t, z_{t}+y_{t}\right)}, \int_{0}^{t} e\left(t, s, z_{\varrho\left(s, z_{s}+y_{s}\right)}+\right.\right.$ $\left.y_{\varrho\left(s, z_{s}+y_{s}\right)} d s\right)$ such that

$$
\begin{aligned}
h(t)= & \mathscr{S}\left(t, z_{\varrho\left(t, z_{t}+y_{t}\right)}+y_{\varrho\left(t, z_{t}+y_{t}\right)}\right)-\mathcal{S}(t) \mathscr{G}(0, \varsigma(0)) \\
& +\int_{0}^{t} \mathcal{S}(t-s) v(s) d s, \quad \text { for every } t \in \mathscr{I}_{n} .
\end{aligned}
$$

From the hypotheses (H2)(ii) and (H3)(ii), we sustain

$$
\begin{aligned}
& H_{d}\left(\mathscr { F } \left(t, z_{\varrho\left(t, z_{t}+y_{t}\right)}\right.\right. \\
& \left.\quad+y_{\varrho\left(t, z_{t}+y_{t}\right)}, \int_{0}^{t} e\left(t, s, z_{\varrho\left(s, z_{s}+y_{s}\right)}+y_{\varrho\left(s, z_{s}+y_{s}\right)}\right) d s\right), \\
& \quad \mathscr{F}\left(t, \bar{z}_{\varrho\left(t, \bar{z}_{t}+y_{t}\right)}\right. \\
& \left.\left.\quad+y_{\varrho\left(t, \bar{z}_{t}+y_{t}\right)}, \int_{0}^{t} e\left(t, s, \bar{z}_{\varrho\left(s, \bar{z}_{s}+y_{s}\right)}+y_{\varrho\left(s, \bar{z}_{s}+y_{s}\right)}\right) d s\right)\right) \\
& \quad \leq \wp_{n}(t)\left[\left\|z_{\varrho\left(t, z_{t}+y_{t}\right)}-\bar{z}_{\varrho\left(t, \bar{z}_{t}+y_{t}\right)}\right\|_{\mathscr{B}}+C_{1} \| z_{\varrho\left(t, z_{t}+y_{t}\right)}\right. \\
& \left.\quad-\bar{z}_{\varrho\left(t, \bar{z}_{t}+y_{t}\right)} \|_{\mathscr{B}}\right] .
\end{aligned}
$$

As a result, there is $w \in \mathscr{F}\left(t, \bar{z}_{\varrho\left(t, \bar{z}_{t}+y_{t}\right)}+y_{\varrho\left(t, \bar{z}_{t}+y_{t}\right)}, \int_{0}^{t} e(t, s\right.$, $\left.\left.\bar{z}_{\varrho\left(s, \bar{z}_{s}+y_{s}\right)}+y_{\varrho\left(s, \bar{z}_{s}+y_{s}\right)}\right) d s\right)$ so that

$$
\begin{aligned}
& |v(t)-w| \leq \wp_{n}(t)\left[\left\|z_{\varrho\left(t, z_{t}+y_{t}\right)}-\bar{z}_{\varrho\left(t, \bar{z}_{t}+y_{t}\right)}\right\|_{\mathscr{B}}\right. \\
& \left.\quad+C_{1}\left\|z_{\varrho\left(t, z_{t}+y_{t}\right)}-\bar{z}_{\varrho\left(t, \bar{z}_{t}+y_{t}\right)}\right\|_{\mathscr{B}}\right] .
\end{aligned}
$$

Recognize $\mathscr{U}_{*}:[0, n] \rightarrow \mathscr{P}(\mathbb{E})$ specified by

$$
\begin{gathered}
\mathcal{U}_{*}(t)=\left\{w \in \mathbb{E}:|v(t)-w| \leq \wp_{n}(t)\right. \\
\cdot\left[\left\|z_{\varrho\left(t, z_{t}+y_{t}\right)}-\bar{z}_{\varrho\left(t, \bar{z}_{t}+y_{t}\right)}\right\|_{\mathscr{B}}\right. \\
\left.\left.+C_{1}\left\|z_{\varrho\left(t, z_{t}+y_{t}\right)}-\bar{z}_{\varrho\left(t, \bar{z}_{t}+y_{t}\right)}\right\|_{\mathscr{B}}\right]\right\} .
\end{gathered}
$$


Considering the fact that the multivalued operator $V_{*}(t)=$ $\mathcal{U}_{*}(t) \cap \mathscr{F}\left(t, \bar{z}_{\varrho\left(t, \bar{z}_{t}+y_{t}\right)}+y_{\varrho\left(t, \bar{z}_{t}+y_{t}\right)}, \int_{0}^{t} e\left(t, s, \bar{z}_{\varrho\left(s, \bar{z}_{s}+y_{s}\right)}+\right.\right.$ $\left.y_{\rho\left(s, \bar{z}_{s}+y_{s}\right)}\right) d s$ ) is measurable (see Proposition 3 ), there is function $\bar{v}(t)$, which is a measurable choice for $V_{*}$. So, $\bar{v}(t) \in$ $\mathscr{F}\left(t, \bar{z}_{\varrho\left(t, \bar{z}_{t}+y_{t}\right)}+y_{\varrho\left(t, \bar{z}_{t}+y_{t}\right)}, \int_{0}^{t} e\left(t, s, \bar{z}_{\varrho\left(s, \bar{z}_{s}+y_{s}\right)}+y_{\varrho\left(s, \bar{z}_{s}+y_{s}\right)}\right) d s\right)$, and, from Remark 9(ii), we specify

$$
|v(t)-\bar{v}(t)| \leq \wp_{n}(t)\left(1+C_{1}\right) \mathscr{D}_{1}^{*}|z(t)-\bar{z}(t)| .
$$

For every $t \in[0, n]$, give us a chance to characterize

$$
\begin{aligned}
\bar{h}(t)= & \mathscr{G}\left(t, \bar{z}_{\varrho\left(t, \bar{z}_{t}+y_{t}\right)}+y_{\varrho\left(t, \bar{z}_{t}+y_{t}\right)}\right)-\mathcal{S}(t) \mathscr{G}(0, \varsigma(0)) \\
& +\int_{0}^{t} \mathcal{S}(t-s) \bar{v}(s) d s
\end{aligned}
$$

Again, from Remark 9(iii), we now have

$$
\begin{aligned}
& |h(t)-\bar{h}(t)| \leq \mid \mathscr{G}\left(t, z_{\varrho\left(t, z_{t}+y_{t}\right)}+y_{\varrho\left(t, z_{t}+y_{t}\right)}\right) \\
& \quad-\mathscr{G}\left(t, \bar{z}_{\varrho\left(t, \bar{z}_{t}+y_{t}\right)}+y_{\varrho\left(t, \bar{z}_{t}+y_{t}\right)}\right) \mid \\
& \quad+\int_{0}^{t}\|\mathcal{S}(t-s)\|_{B(\mathbb{E})}|v(s)-\bar{v}(s)| d s \leq\left(\wp_{n}^{*}\right. \\
& \left.\quad+\frac{1}{\tau}\right) e^{\tau L_{n}^{*}(t)}\|z-\bar{z}\|_{n} .
\end{aligned}
$$

As a result

$$
\|h-\bar{h}\|_{n} \leq\left(\wp_{n}^{*}+\frac{1}{\tau}\right)\|z-\bar{z}\|_{n} .
$$

Being practically equivalent to the connection gotten by exchanging the parts of $z$ and $\bar{z}$, it takes after that

$$
H_{d}(\bar{\Upsilon}(z), \bar{\Upsilon}(\bar{z})) \leq\left(\wp_{n}^{*}+\frac{1}{\tau}\right)\|z-\bar{z}\|_{n},
$$

demonstrating that $\bar{\Upsilon}$ is a contraction for all $n \in \mathbb{N}$ and, from the second aspect of [30, Theorem 3.4], we realize that $\bar{\Upsilon}$ is likewise admissible contraction operator. With the decision of $\mathcal{U}$, there is no $z \in \partial \mathcal{U}$ in a way that $z=\lambda \bar{\Upsilon}(z)$ for many $\lambda \in(0,1)$. From the nonlinear alternative fixed point theorem thanks to Frigon [1, Corollary 3.5], we realize that the operator $\bar{\Upsilon}$ has a fixed point $z^{*}$. This intimates that $x^{*}(t)=z^{*}(t)+y(t)$, $t \in(-\infty,+\infty)$, is a fixed point of the operator $\Upsilon$, which is a mild solution of the structure (1).

\section{Illustrative Example}

To exemplify our theoretical results, we treat the FNIDI with SDD of the structure

$$
\begin{gathered}
\frac{\partial}{\partial t}[u(t, \xi)-g(t, u(t-\sigma(u(t, 0)), \xi))] \\
\in \int_{t}^{0} \frac{(t-s)^{\alpha-2}}{\Gamma(\alpha-1)}\left(\frac{\partial^{2}}{\partial \xi^{2}}-r\right)[u(s, \xi)
\end{gathered}
$$

$$
\begin{aligned}
& -g(s, u(s-\sigma(u(s, 0)), \xi))] d s+\left[f_{1}(t,\right. \\
& u(t-\sigma(u(t, 0)), \xi), \\
& \left.\int_{0}^{t} \eta(t, s, u(s-\sigma(u(s, 0)), \xi)) d s\right), f_{2}(t, \\
& u(t-\sigma(u(t, 0)), \xi), \\
& \left.\left.\int_{0}^{t} \eta(t, s, u(s-\sigma(u(s, 0)), \xi)) d s\right)\right], \\
& u(t, 0)=u(t, \pi)=0, \quad t \in[0, \infty), \\
& u(\theta, \xi)=u_{0}(\theta, \xi), \quad \theta \in(-\infty, 0], \xi \in[0, \pi],
\end{aligned}
$$

where $1<\alpha<2,\left(u_{0}, \sigma\right) \in C(\mathbb{R}[0, \infty)), L_{\xi}=\left(\partial^{2} / \partial \xi^{2}-\right.$ $r), r>0$ stands for the operator with respect to the special variable $\xi, f_{1}, f_{2}: \mathscr{I} \times \mathscr{B} \times \mathbb{E} \rightarrow \mathbb{R}$ are measurable in $t$ and continuous in $x, g: \mathscr{I} \times \mathscr{B} \rightarrow \mathbb{R}$, and $\eta: \mathscr{I} \times \mathscr{I} \times \mathscr{B} \rightarrow$ $\mathbb{R}$ are appropriate functions. We expect that, for each $t \geq 0$, $f_{1}(t, \cdot, \cdot)$ is lower semicontinuous (i.e., the set $\{x \in \mathscr{B}, y \in \mathbb{E}$ : $\left.f_{1}(t, x, y)>v\right\}$ is open for all $\left.\nu \in \mathbb{R}\right)$ and accept that, for each $t \geq 0, f_{2}(t, \cdot, \cdot)$ is upper semicontinuous (i.e., the set $\{x \in \mathscr{B}$, $\left.y \in \mathbb{E}: f_{2}(t, x, y)<\nu\right\}$ is open for each $\left.\nu \in \mathbb{R}\right)$.

Recognize $\mathbb{E}=L^{2}([0, \pi], \mathbb{R})$ and the operator $\mathscr{A}: L_{\xi}$ : $D(\mathscr{A}) \subset \mathbb{E} \rightarrow \mathbb{E}$ with domain

$$
D(\mathscr{A})=\left\{u \in \mathbb{E}: u^{\prime \prime} \in \mathbb{E}, u(0)=u(\pi)=0\right\} .
$$

Clearly, $\mathscr{A}$ is densely defined in $\mathbb{E}$ and is sectorial. Hence, $\mathscr{A}$ is a generator of a solution operator on $\mathbb{E}$. For the phase space, we pick $\mathscr{B}=C_{\gamma}=\left\{\varsigma \in C((-\infty, 0]: \mathbb{X}): \lim _{\theta \rightarrow-\infty} e^{\gamma \theta} \varsigma(\theta)\right.$ exists in $\mathbb{X}$ \} invested with the norm

$$
|\varsigma|=\sup _{-\infty<\theta \leq 0} e^{\gamma \theta}|\varsigma(\theta)| .
$$

Here, we note the phase space $C_{\gamma}$ satisfying conditions $\left(P_{1}\right)$, $\left(P_{2}\right)$, and $\left(P_{3}\right)$. Set

$$
\begin{aligned}
& x(t)(\xi)=u(t, \xi), \quad t \geq 0, \xi \in[0, \pi], \\
& \varsigma(0)(\xi)=u_{0}(\theta, \xi), \quad t \geq 0, \theta \leq 0, \\
& \mathscr{G}(t, \varsigma)(\xi)=g(t, \varsigma(0, \xi)), \quad t \geq 0, \xi \in[0, \pi], \\
& \mathscr{F}(t, \varsigma, x)(\xi) \\
& \quad=\left[f_{1}\left(t, \varsigma(0, \xi), \int_{0}^{t} \eta(t, s, \varsigma(0, \xi)) d s\right),\right. \\
& \left.\quad f_{2}\left(t, \varsigma(0, \xi), \int_{0}^{t} \eta(t, s, \varsigma(0, \xi)) d s\right)\right], \\
& e(t, s, \varsigma)(\xi)=\eta(t, s, \varsigma(0, \xi)), \\
& \varrho(t, \varsigma)=t-\sigma(\varsigma(0,0)) .
\end{aligned}
$$


The multivalued map $\mathscr{F}$ is u.s.c. with compact convex values. Thus, (H1) and (H2) are fulfilled.

Presently, the existence of mild solutions can be reasoned from an immediate utilization of Theorem 8 . In the perspective of phase space $C_{\gamma}$ with the above examination, we have the accompanying results.

Corollary 10. Let $\varsigma \in \mathscr{B}=C_{\gamma}$ be continuous and bounded. Then, there is certainly at least one mild solution of the model (57) on $(-\infty,+\infty)$.

\section{Conflict of Interests}

The authors declare that there is no conflict of interests regarding the publication of this paper.

\section{Acknowledgment}

The fourth author would like to dedicate this paper, with love and respect, to his doctoral supervisor Professor A. Anguraj, P.S.G. College of Arts \& Science, Coimbatore 641014, India, on the occasion of his 54 th birthday.

\section{References}

[1] M. Frigon, "Fixed point results for multivalued contractions on gauge spaces," in Set Valued Mappings with Applications in Nonlinear Analysis, R. P. Agarwal and D. O’Regan, Eds., vol. 4 of Series in Mathematical Analysis and Applications, pp. 175-181, Taylor \& Francis, London, UK, 2002.

[2] C. Cuevas and J. C. de Souza, "Existence of $S$-asymptotically $\omega$-periodic solutions for fractional order functional integrodifferential equations with infinite delay," Nonlinear Analysis: Theory, Methods \& Applications, vol. 72, no. 3-4, pp. 1683-1689, 2010.

[3] S. Abbas, M. Benchohra, and G. M. N'Guerekata, Topics in Fractional Differential Equations, Springer, New York, NY, USA, 2012.

[4] D. Baleanu, J. A. T. Machado, and A. C. J. Luo, Fractional Dynamics and Control, Springer, New York, NY, USA, 2012.

[5] I. Podlubny, Fractional Differential Equations, Academic Press, New York, NY, USA, 1999.

[6] K. Diethelm, The Analysis of Fractional Differential Equations, Springer, Berlin, Germany, 2010.

[7] A. A. Kilbas, H. M. Srivastava, and J. J. Trujillo, Theory and Applications of Fractional Differential Equations, Elsevier, Amsterdam, The Netherlands, 2006.

[8] Y.Zhou, Basic Theory of Fractional Differential Equations, World Scientific, Singapore, 2014.

[9] J. Li and J. Qi, "Eigenvalue problems for fractional differential equations with right and left fractional derivatives," Applied Mathematics and Computation, vol. 256, pp. 1-10, 2015.

[10] J. Ren, Z.-Z. Sun, and W. Dai, "New approximations for solving the Caputo-type fractional partial differential equations," Applied Mathematical Modelling, 2015.

[11] X.-B. Shu, F. Xu, and Y. Shi, "S-asymptotically $\omega$-positive periodic solutions for a class of neutral fractional differential equations," Applied Mathematics and Computation, vol. 270, pp. 768-776, 2015.
[12] M. Yi, L. Wang, and H. Jun, "Legendre wavelets method for the numerical solution of fractional integro-differential equations with weakly singular kernel," Applied Mathematical Modelling, 2015.

[13] A. Chadha and D. N. Pandey, "Existence results for an impulsive neutral fractional integrodifferential equation with infinite delay," International Journal of Differential Equations, vol. 2014, Article ID 780636, 10 pages, 2014.

[14] J. Wang, A. G. Ibrahim, and M. Fečkan, "Nonlocal impulsive fractional differential inclusions with fractional sectorial operators on Banach spaces," Applied Mathematics and Computation, vol. 257, pp. 103-118, 2015.

[15] S. Suganya, M. M. Arjunan, and J. J. Trujillo, "Existence results for an impulsive fractional integro-differential equation with state-dependent delay," Applied Mathematics and Computation, vol. 266, no. 1, pp. 54-69, 2015.

[16] Z. Liu and B. Zeng, "Existence and controllability for fractional evolution inclusions of Clarke's subdifferential type," Applied Mathematics and Computation, vol. 257, pp. 178-189, 2015.

[17] Z. Yan and X. Jia, "Approximate controllability of partial fractional neutral stochastic functional integro-differential inclusions with state-dependent delay," Collectanea Mathematica, vol. 66, no. 1, pp. 93-124, 2015.

[18] P. Balasubramaniam and P. Tamilalagan, "Approximate controllability of a class of fractional neutral stochastic integrodifferential inclusions with infinite delay by using Mainardi's function," Applied Mathematics and Computation, vol. 256, pp. 232-246, 2015.

[19] A. Anguraj, M. Mallika Arjunan, and E. Hernández, "Existence results for an impulsive neutral functional differential equation with state-dependent delay," Applicable Analysis, vol. 86, no. 7, pp. 861-872, 2007.

[20] M. Benchohra and I. Medjadj, "Global existence results for neutral functional differential equations with state-dependent delay ", Differential Equations and Dynamical Systems, 2014.

[21] M. E. Hernández and M. A. McKibben, "On state-dependent delay partial neutral functional-differential equations," Applied Mathematics and Computation, vol. 186, no. 1, pp. 294-301, 2007.

[22] M. M. Arjunan and V. Kavitha, "Existence results for impulsive neutral functional differential equations with state-dependent delay," Electronic Journal of Qualitative Theory of Differential Equations, no. 26, pp. 1-13, 2009.

[23] G. R. Gautam and J. Dabas, "Mild solutions for class of neutral fractional functional differential equations with not instantaneous impulses," Applied Mathematics and Computation, vol. 259, pp. 480-489, 2015.

[24] M. Benchohra and S. Litimein, "Functional integro-differential equations with statedependent delay in Fréchet spaces," Memoirs on Differential Equations and Mathematical Physics, vol. 61, pp. 21-36, 2014.

[25] Y.-K. Chang, M. M. Arjunan, and V. Kavitha, "Existence results for neutral functional integrodifferential equations with infinite delay via fractional operators," Journal of Applied Mathematics and Computing, vol. 36, no. 1-2, pp. 201-218, 2011.

[26] R. P. Agarwal, B. de Andrade, and G. Siracusa, "On fractional integro-differential equations with state-dependent delay," Computers \& Mathematics with Applications, vol. 62, no. 3, pp. 1143-1149, 2011.

[27] M. Benchohra, S. Litimein, and G. N'Guerekata, “On fractional integro-differential inclusions with state-dependent delay in Banach spaces," Applicable Analysis, vol. 92, no. 2, pp. 335-350, 2013. 
[28] M. Benchohra, S. Litimein, J. J. Trujillo, and M. P. Valasco, "Abstract fractional integro-differential equations with statedependent delay," International Journal of Evolution Equations, vol. 6, no. 2, pp. 115-128, 2011.

[29] M. Benchohra and S. Litimein, "Fractional integro-differential equations with state-dependent delay on an unbounded domain," African Diaspora Journal of Mathematics, vol. 12, no. 2, pp. 13-25, 2011.

[30] M. Benchohra and S. Litimein, "The existence and controllability results for fractional order integro-differential inclusions in Fréchet spaces," Proceedings of A. Razmadze Mathematical Institute, vol. 162, pp. 1-23, 2013.

[31] K. Yosida, Functional Analysis, Springer, Berlin, Germany, 6th edition, 1980.

[32] J. K. Hale and J. Kato, "Phase space for retarded equations with infinite delay," Funkcialaj Ekvacioj, vol. 21, no. 1, pp. 11-41, 1978.

[33] Y. Hino, S. Murakami, and T. Naito, Functional Differential Equations with Unbounded Delay, Springer, Berlin, Germany, 1991.

[34] J. K. Hale and S. M. Verduyn Lunel, Introduction to FunctionalDifferential Equations: Applied Mathematical Sciences, vol. 99, Springer, New York, NY, USA, 1993.

[35] C. Lizama, "On approximation and representation of $k$ regularized resolvent families," Integral Equations and Operator Theory, vol. 41, no. 2, pp. 223-229, 2001.

[36] J. Pruss, Evolutionary Integral Equations and Applications, Monographs in Mathematics, vol. 87, Birkhäuser, Basel, Switzerland, 1993.

[37] C. Castaing and M. Valadier, Convex Analysis and Measurable Multifunctions, vol. 580 of Lecture Notes in Mathematics, Springer, Berlin, Germany, 1977.

[38] J. R. Graef, J. Henderson, and A. Ouahab, Impulsive Differential Inclusions: A Fixed Point Approach, Walter de Gruyter, Berlin, Germany, 2013. 


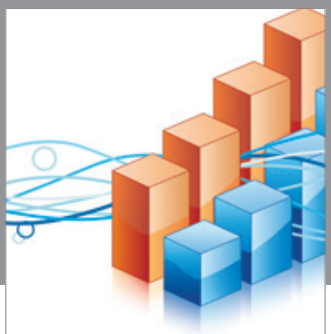

Advances in

Operations Research

vatem alat4

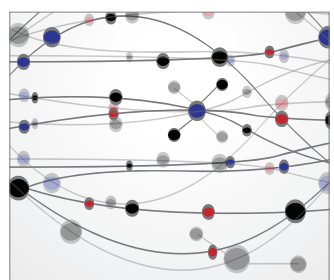

\section{The Scientific} World Journal
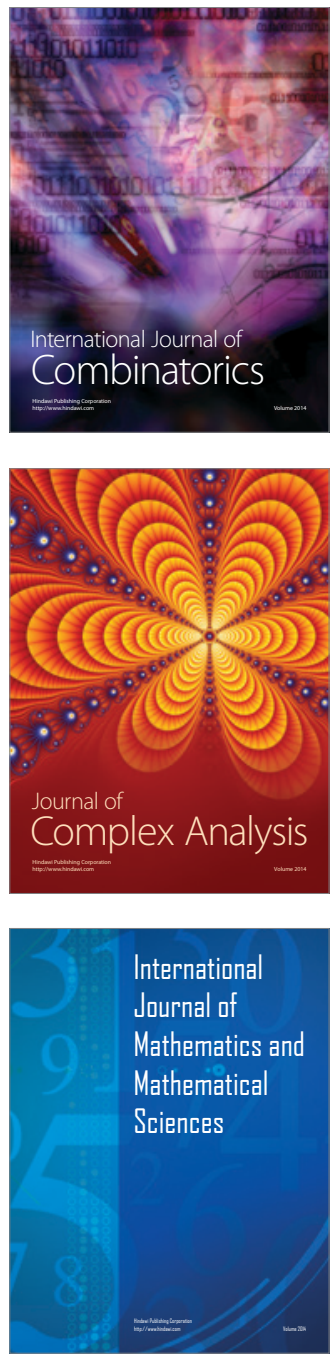
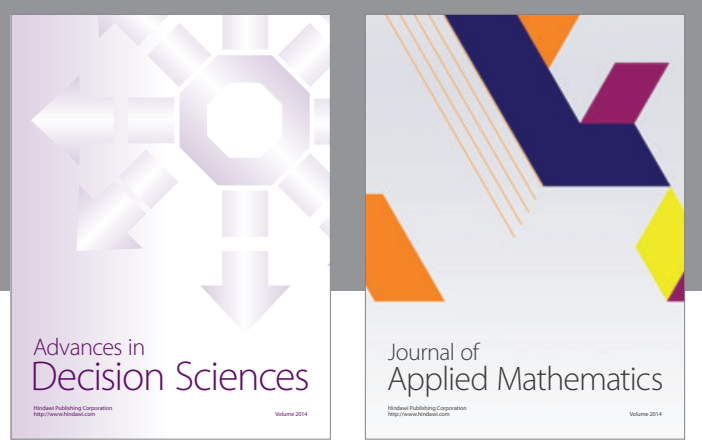

Algebra

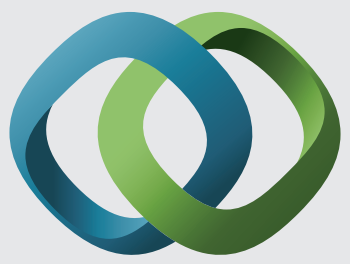

\section{Hindawi}

Submit your manuscripts at

http://www.hindawi.com
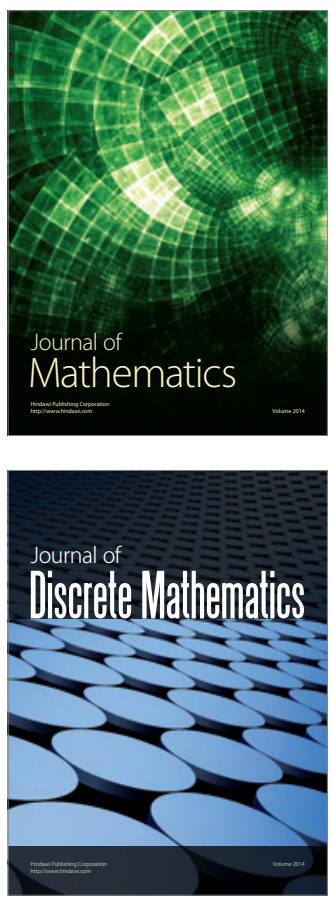

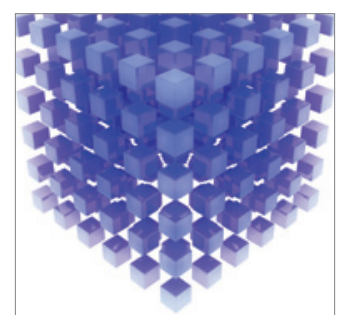

Mathematical Problems in Engineering
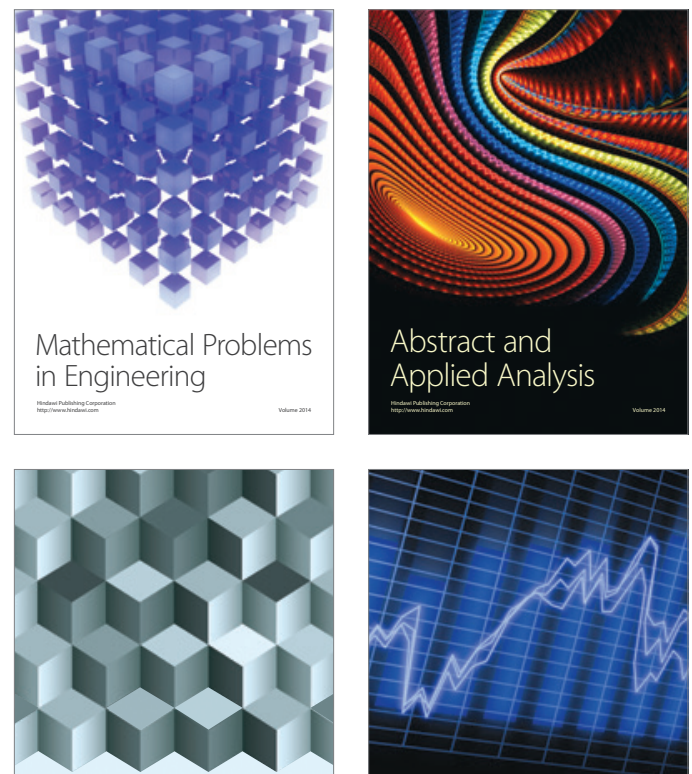

Journal of

Function Spaces

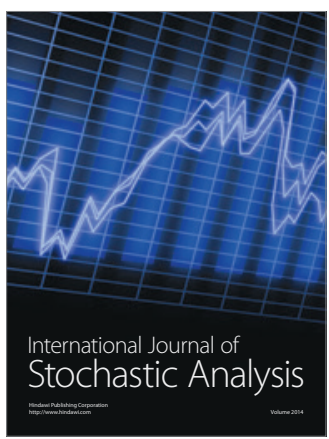

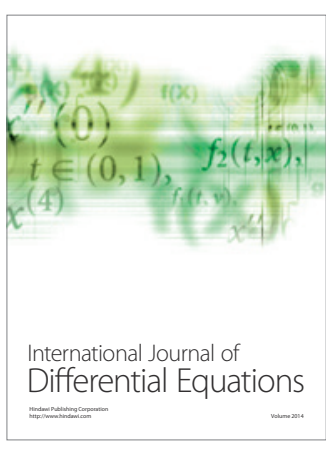
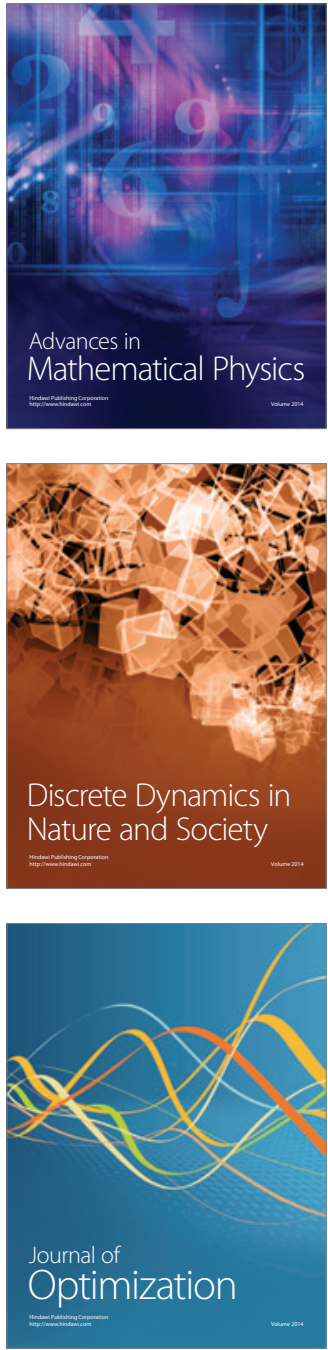